\title{
DOI: https://doi.org/10.24297/jam.v19i.8800
}

\section{Modified New Iterative Method for Solving Nonlinear Partial Differential Equations}

\author{
Alaa K. Jabber ${ }^{1}$ \\ ${ }^{1}$ Department of Mathematics, College of Education, University of Al-Qadisiyah, Iraq
}

\begin{abstract}
In this paper, the iterative method, proposed by Gejji and Jafari in 2006, has been modified for solving nonlinear initial value problems. The Laplace transform was used in this modification to eliminate the linear differential operator in the differential equation. The convergence of the solution was discussed according to the modification proposed. To illustrate this modification, some examples were presented.
\end{abstract}

Keywords: Iterative Method, partial differential equations, initial value problem, numerical solution, Laplace transform.

\section{Introduction}

Differential equations appear in many scientific and life fields such as engineering, physics, chemistry, economics, and biology. Therefore, it became necessary to focus on finding the best methods to solve it. There are many numerical and analytical methods used to solve partial differential equations, such as Admoain decomposition method [1 - 6], homotopy perturbation method [7 - 11], Laplace decomposition method [12, 13], variational iteration method [14 - 16], collocation method [17-19] and artificial neural network (Ann) [20 24].

In 2006, Gejji and Jafari proposed a new iterative method (NIM) for solving functional equations [25]. They described the general functional equation as following:

$$
u=N(u)+f
$$

where $N$ is a nonlinear operator and $f$ is a known function. The solution $u$ expressed as the form:

$$
u=\sum_{m=0}^{\infty} u_{m}
$$

They decomposed the nonlinear operator $N$ as:

$$
N\left(\sum_{m=0}^{\infty} u_{m}\right)=N\left(u_{0}\right)+\sum_{m=1}^{\infty}\left\{N\left(\sum_{j=0}^{m} u_{j}\right)-N\left(\sum_{j=0}^{m-1} u_{j}\right)\right\}
$$

From (2) and (3), equation (1) is equivalent to

$$
\sum_{m=0}^{\infty} u_{m}=f+N\left(u_{0}\right)+\sum_{m=1}^{\infty}\left\{N\left(\sum_{j=0}^{m} u_{j}\right)-N\left(\sum_{j=0}^{m-1} u_{j}\right)\right\}
$$

Finally, the recurrence relation was defined as:

$$
\left\{\begin{array}{l}
u_{0}=f \\
u_{1}=N\left(u_{0}\right) \\
u_{m+1}=N\left(u_{0}+\cdots+u_{m}\right)-N\left(u_{0}+\cdots+u_{m-1}\right), m=1,2, \ldots
\end{array}\right.
$$

Later, NIM was used and modified to solve nonlinear equations such as partial differential equations of integer and fractional order, integral equations, a system of equations, and algebraic equations [26-30].

In this paper, the NIM was modified to solve partial differential equations. The Laplace transform was used to convert the partial differential equation into a formula similar to the formula (1) as it will be illustrated in the next section. 


\section{Modified New Iterative Method}

To illustrate the modified method, rewrite a general IVP as:

$$
L(u(X, t))+R(u(X, t))+N(u(X, t))=g(X, t)
$$

With the initial condition

$$
\left.\frac{\partial^{k} u(X, t)}{\partial t^{k}}\right|_{t=0}=f_{k}(X), k=0,1, \ldots, n-1
$$

where $L()=.\frac{\partial^{n}(.)}{\partial t^{n}}, n=1,2,3, \ldots$ is a linear operator of the partial derivative with respect to $t, R($.$) the remained$ of the linear operator, $N($.$) the nonlinear operator, g(X, t)$ is the inhomogeneous part which is known function and $X$ is a variable with one or more dimensions.

Taking the Laplace transform (with respect to the variable $t$ ) for the equation (6) to get:

$$
\mathcal{L}\{L(u)\}+\mathcal{L}\{R(u)+N(u)\}=\mathcal{L}\{g\}
$$

By the properties of the Laplace transform and put $\mathcal{F}=R+N$, the equation (6) becomes:

$$
s^{n} \mathcal{L}\{u\}-\left.\sum_{i=0}^{n-1} s^{n-i-1} \frac{\partial^{i} u}{\partial t^{i}}\right|_{t=0}+\mathcal{L}\{\mathcal{F}(u)\}=\mathcal{L}\{g\}
$$

From (7) we have:

$$
s^{n} \mathcal{L}\{u\}-\sum_{i=0}^{n-1} s^{n-i-1} f_{i}+\mathcal{L}\{\mathcal{F}(u)\}=\mathcal{L}\{g\}
$$

And hence:

$$
\mathcal{L}\{u\}=\sum_{i=0}^{n-1} s^{-i-1} f_{i}+\frac{1}{s^{n}} \mathcal{L}\{g\}-\frac{1}{s^{n}} \mathcal{L}\{\mathcal{F}(u)\}
$$

Taking the inverse of the Laplace transform on both sides of equation (11), to get:

$$
u=\sum_{i=0}^{n-1} f_{i} \frac{t^{i}}{i !}+\mathcal{L}^{-1}\left\{\frac{1}{s^{n}} \mathcal{L}\{g\}\right\}-\mathcal{L}^{-1}\left\{\frac{1}{s^{n}} \mathcal{L}\{\mathcal{F}(u)\}\right\}
$$

By the linearity property of the Laplace transform and it's inverse:

$$
u=\sum_{i=0}^{n-1} f_{i} \frac{t^{i}}{i !}+\mathcal{L}^{-1}\left\{\frac{1}{s^{n}} \mathcal{L}\{g\}\right\}-\mathcal{L}^{-1}\left\{\frac{1}{s^{n}} \mathcal{L}\{\mathcal{F}(u)\}\right\}
$$

Then (13) is equivalent to (6), i.e. the two equations have the same solutions.

Substituting (2) in (13) we have:

$$
\sum_{m=0}^{\infty} u_{m}(X, t)=f-\mathcal{L}^{-1}\left\{\frac{1}{s^{n}} \mathcal{L}\left\{\mathcal{F}\left(\sum_{m=0}^{\infty} u_{m}(X, t)\right)\right\}\right\}
$$

Where

$$
f=\sum_{i=0}^{n-1} f_{i} \frac{t^{i}}{i !}+\mathcal{L}^{-1}\left\{\frac{1}{s^{n}} \mathcal{L}\{g\}\right\}
$$

The operator $\mathcal{F}$ in (14) can be decomposed as: 


$$
\mathcal{L}^{-1}\left\{\frac{1}{s^{n}} \mathcal{L}\left\{\mathcal{F}\left(\sum_{m=0}^{\infty} u_{m}\right)\right\}\right\}=\mathcal{L}^{-1}\left\{\frac{1}{s^{n}} \mathcal{L}\left\{\mathcal{F}\left(u_{0}\right)+\sum_{m=1}^{\infty}\left[\mathcal{F}\left(\sum_{j=0}^{m} u_{j}\right)-\mathcal{F}\left(\sum_{j=0}^{m-1} u_{j}\right)\right]\right\}\right\}
$$

Substituting (16) in (14), we have:

$$
\sum_{m=0}^{\infty} u_{m}=f-\mathcal{L}^{-1}\left\{\frac{1}{s^{n}} \mathcal{L}\left\{\mathcal{F}\left(u_{0}\right)+\sum_{i=1}^{\infty}\left[\mathcal{F}\left(\sum_{j=0}^{m} u_{j}\right)-\mathcal{F}\left(\sum_{j=0}^{m-1} u_{j}\right)\right]\right\}\right\}
$$

Then, compared to the NIM (1-5), the recurrence relation can be defined as follows:

$$
\left\{\begin{array}{l}
u_{0}=f \\
u_{1}=-\mathcal{L}^{-1}\left\{\frac{1}{s^{n}} \mathcal{L}\left\{\mathcal{F}\left(u_{0}\right)\right\}\right\} \\
u_{m+1}=-\mathcal{L}^{-1}\left\{\frac{1}{s^{n}} \mathcal{L}\left\{\mathcal{F}\left(u_{0}+\cdots+u_{m}\right)-\mathcal{F}\left(u_{0}+\cdots+u_{m-1}\right)\right\}\right\}, \quad m=1,2, \ldots
\end{array}\right.
$$

\section{Convergence analysis}

In this section, the convergence of the series (2), calculated by (18), will be discussed, and it will also be proved that it satisfies the initial value problem (6). But first, the following three lemmas will be presented.

Lemma 1: If $L^{-1}(F)$ and $L^{-1}(\|F\|)$ are exist, where $L$ is the Laplace transform, for any function $F$ in a Banach space $B$, then

$$
\left\|L^{-1}(F)\right\|=L^{-1}(\|F\|)
$$

\section{Proof:}

The Post-Widder inversion formula for the Laplace transform is: [31]

$$
L^{-1}(F)=\lim _{k \rightarrow \infty}\left[\frac{(-1)^{k}}{k !}\left(\frac{k}{t}\right)^{k+1} F^{(k)}\left(\frac{k}{t}\right)\right], \quad t>0 \text { and } k=0,1,2, \ldots
$$

Since $L^{-1}(F)$ is exist then there is $f(t)$ such that

$$
L^{-1}(F)=\lim _{k \rightarrow \infty}\left[\frac{(-1)^{k}}{k !}\left(\frac{k}{t}\right)^{k+1} F^{(k)}\left(\frac{k}{t}\right)\right]=f(t)
$$

Then

$$
\begin{aligned}
\|f(t)\|=\left\|L^{-1}(f)\right\| & =\left\|\lim _{k \rightarrow \infty}\left[\frac{(-1)^{k}}{k !}\left(\frac{k}{t}\right)^{k+1} F^{(k)}\left(\frac{k}{t}\right)\right]\right\|=\lim _{k \rightarrow \infty}\left[\left\|\frac{1}{k !}\left(\frac{k}{t}\right)^{k+1} F^{(k)}\left(\frac{k}{t}\right)\right\|\right] \\
= & \lim _{k \rightarrow \infty}\left[\left\|\frac{(-1)^{k}}{k !}\right\|\left\|\left(\frac{k}{t}\right)^{k+1}\right\|\left\|F^{(k)}\left(\frac{k}{t}\right)\right\|\right]=\lim _{k \rightarrow \infty}\left[\frac{1}{k !}\left(\frac{k}{t}\right)^{k+1}\left\|F^{(k)}\left(\frac{k}{t}\right)\right\|\right]
\end{aligned}
$$

Then the sequence $\left\{\frac{1}{k !}\left(\frac{k}{t}\right)^{k+1}\left\|F^{(k)}\left(\frac{k}{t}\right)\right\|\right\}$ converges to $\|f(t)\|$.

Now since $L^{-1}(\|F\|)$ is exist then there is $\beta$ such that $L^{-1}(\|F\|)=\beta$ i.e. by the Post-Widder inversion formula for the Laplace transform we have

$$
L^{-1}(\|F\|)=\lim _{k \rightarrow \infty}\left[\frac{(-1)^{k}}{k !}\left(\frac{k}{t}\right)^{k+1}\left\|F^{(k)}\left(\frac{k}{t}\right)\right\|\right]=\beta
$$

Then the sequence $\left\{\frac{(-1)^{k}}{k !}\left(\frac{k}{t}\right)^{k+1}\left\|F^{(k)}\left(\frac{k}{t}\right)\right\|\right\}$ converges to $\beta$. But this sequence has a subsequence $\left\{\frac{1}{k !}\left(\frac{k}{t}\right)^{k+1}\left\|F^{(k)}\left(\frac{k}{t}\right)\right\|\right\}$ which is converging to $\|f(t)\|$, and every convergent subsequence of a convergent sequence has the same convergence point, that implies $\beta=\|f(t)\|$ and hence $L^{-1}(\|F\|)=\|f(t)\|$, then 


$$
\left\|L^{-1}(F)\right\|=L^{-1}(\|F\|)
$$

Lemma 2: If $L(f)$ and $L(\|f\|)$ are exist, where $L$ is the Laplace transform, for any function $f$ in a Banach space $B$, then

$$
\|L(f)\| \leq L(\|f\|)
$$

\section{Proof:}

The Laplace transform is defined as:

$$
L(f)=\int_{0}^{\infty} e^{-s t} f(t) d t
$$

Therefore,

$$
\|L(f)\|=\left\|\int_{0}^{\infty} e^{-s t} f(t) d t\right\| \leq \int_{0}^{\infty}\left\|e^{-s t} f(t)\right\| d t=\int_{0}^{\infty} e^{-s t}\|f(t)\| d t=L(\|f\|)
$$

Then

$$
\|L(f)\| \leq L(\|f\|)
$$

Lemma 3: For any function $f$ in a Banach space $B$

$$
\left\|L^{-1}\left[\frac{1}{s^{n}} L(f)\right]\right\| \leq L^{-1}\left[\frac{1}{s^{n}} L(\|f\|)\right] \quad, s>0, n=1,2, \ldots
$$

\section{Proof:}

By Lemma 1 and Lemma 2 and since $s>0$, we have

$$
\left\|L^{-1}\left[\frac{1}{s^{n}} L(f)\right]\right\|=L^{-1}\left[\left\|\frac{1}{s^{n}} L(f)\right\|\right]=L^{-1}\left[\frac{1}{s^{n}}\|L(f)\|\right] \leq L^{-1}\left[\frac{1}{s^{n}} L(\|f\|)\right]
$$

Theorem 1: If $\mathcal{F}$ is a contraction operator, i.e., there is $\alpha \in(0,1)$ such that $\|\mathcal{F}(u(t, X))-\mathcal{F}(v(t, X))\| \leq$ $\alpha\|u(t, X)-v(t, X)\|, t \geq 0$. Then, the infinite series (2), which is computed by (18), is absolutely convergent if $t<\sqrt[n]{n ! / \alpha}$.

\section{Proof:}

For any $m=1,2,3, \ldots$, by $(18)$ :

$$
\left\|u_{m+1}\right\|=\left\|\mathcal{L}^{-1}\left\{\frac{1}{s^{n}} \mathcal{L}\left\{\mathcal{F}\left(u_{0}+\cdots+u_{m}\right)-\mathcal{F}\left(u_{0}+\cdots+u_{m-1}\right)\right\}\right\}\right\|
$$

By Lemma 3 we have:

$$
\begin{aligned}
\left\|u_{m+1}\right\|=\| \mathcal{L}^{-1} & \left\{\frac{1}{s^{n}} \mathcal{L}\left\{\mathcal{F}\left(u_{0}+\cdots+u_{m}\right)-\mathcal{F}\left(u_{0}+\cdots+u_{m-1}\right)\right\}\right\} \| \\
& \leq \mathcal{L}^{-1}\left\{\frac{1}{s^{n}} \mathcal{L}\left\{\left\|\mathcal{F}\left(u_{0}+\cdots+u_{m}\right)-\mathcal{F}\left(u_{0}+\cdots+u_{m-1}\right)\right\|\right\}\right\} \\
& \leq \mathcal{L}^{-1}\left\{\frac{1}{s^{n}} \mathcal{L}\left\{\alpha\left\|\left(u_{0}+\cdots+u_{m}\right)-\left(u_{0}+\cdots+u_{m-1}\right)\right\|\right\}\right\}=\mathcal{L}^{-1}\left\{\frac{1}{s^{n}} \mathcal{L}\left\{\alpha\left\|u_{m}\right\|\right\}\right\} \\
& =\alpha\left\|u_{m}\right\| \mathcal{L}^{-1}\left\{\frac{1}{s^{n}} \mathcal{L}\{1\}\right\}=\alpha \frac{t^{n}}{n !}\left\|u_{m}\right\|
\end{aligned}
$$

Then

$$
\left\|u_{m+1}\right\| \leq \alpha \frac{t^{n}}{n !}\left\|u_{m}\right\| \quad \rightarrow \quad \frac{\left\|u_{m+1}\right\|}{\left\|u_{m}\right\|} \leq \alpha \frac{t^{n}}{n !} \quad m=1,2,3, \ldots
$$


By the ratio test, the series (2) is convergent if $\frac{\left\|u_{m+1}\right\|}{\left\|u_{m}\right\|}<1$, this implies that

$$
\alpha \frac{t^{n}}{n !}<1 \quad \rightarrow \quad t^{n}<\frac{n !}{\alpha} \quad \rightarrow \quad t<\sqrt[n]{n ! / \alpha}
$$

Theorem 2: The infinite series (2), which is computed by (18), is a solution to (6).

\section{Proof:}

Since $R$ is a linear operator then:

$$
\begin{aligned}
\mathcal{F}\left(u_{0}+\cdots+u_{m}\right) & -\mathcal{F}\left(u_{0}+\cdots+u_{m-1}\right) \\
& =R\left(u_{0}+\cdots+u_{m}\right)+N\left(u_{0}+\cdots+u_{m}\right)-R\left(u_{0}+\cdots+u_{m-1}\right)-N\left(u_{0}+\cdots+u_{m-1}\right) \\
& =R\left(u_{0}\right)+\cdots+R\left(u_{m}\right)-R\left(u_{0}\right)-\cdots-R\left(u_{m-1}\right)+N\left(u_{0}+\cdots+u_{m}\right)-N\left(u_{0}+\cdots+u_{m-1}\right) \\
& =R\left(u_{m}\right)+N\left(u_{0}+\cdots+u_{m}\right)-N\left(u_{0}+\cdots+u_{m-1}\right)
\end{aligned}
$$

Now, Let

$$
\begin{aligned}
S_{m}=u_{1}+\cdots+ & u_{m+1} \\
& =-\mathcal{L}^{-1}\left\{\frac{1}{s^{n}} \mathcal{L}\left\{\mathcal{F}\left(u_{0}\right)\right\}\right\}-\mathcal{L}^{-1}\left\{\frac{1}{s^{n}} \mathcal{L}\left\{\mathcal{F}\left(u_{0}+u_{1}\right)-\mathcal{F}\left(u_{0}\right)\right\}\right\} \\
& -\mathcal{L}^{-1}\left\{\frac{1}{s^{n}} \mathcal{L}\left\{\mathcal{F}\left(u_{0}+u_{1}+u_{2}\right)-\mathcal{F}\left(u_{0}+u_{1}\right)\right\}\right\}-\cdots \\
& -\mathcal{L}^{-1}\left\{\frac{1}{s^{n}} \mathcal{L}\left\{\mathcal{F}\left(u_{0}+\cdots+u_{m}\right)-\mathcal{F}\left(u_{0}+\cdots+u_{m-1}\right)\right\}\right\} \\
& =-\mathcal{L}^{-1}\left\{\frac{1}{s^{n}} \mathcal{L}\left\{R\left(u_{0}\right)+N\left(u_{0}\right)\right\}\right\}-\mathcal{L}^{-1}\left\{\frac{1}{s^{n}} \mathcal{L}\left\{R\left(u_{1}\right)+N\left(u_{0}+u_{1}\right)-N\left(u_{0}\right)\right\}\right\} \\
& -\mathcal{L}^{-1}\left\{\frac{1}{s^{n}} \mathcal{L}\left\{R\left(u_{2}\right)+N\left(u_{0}+u_{1}+u_{2}\right)-N\left(u_{0}+u_{1}\right)\right\}\right\}-\cdots \\
& -\mathcal{L}^{-1}\left\{\frac{1}{s^{n}} \mathcal{L}\left\{R\left(u_{m}\right)+N\left(u_{0}+\cdots+u_{m}\right)-N\left(u_{0}+\cdots+u_{m-1}\right)\right\}\right\} \\
& =-\mathcal{L}^{-1}\left\{\frac{1}{s^{n}} \mathcal{L}\left\{R\left(u_{0}+\cdots+u_{m}\right)+N\left(u_{0}+\cdots+u_{m}\right)\right\}\right\}=-\mathcal{L}^{-1}\left\{\frac{1}{s^{n}} \mathcal{L}\left\{R\left(\sum_{j=0}^{m} u_{j}\right)+N\left(\sum_{j=0}^{m} u_{j}\right)\right\}\right\}
\end{aligned}
$$

Then

$$
\begin{aligned}
u=\sum_{i=0}^{\infty} u_{i}=u_{0} & +\sum_{i=1}^{\infty} u_{i}=f+\lim _{m \rightarrow \infty}\left(S_{m}\right)=f+\lim _{m \rightarrow \infty}\left(-\mathcal{L}^{-1}\left\{\frac{1}{s^{n}} \mathcal{L}\left\{R\left(\sum_{j=0}^{m} u_{j}\right)+N\left(\sum_{j=0}^{m} u_{j}\right)\right\}\right\}\right) \\
& =f-\mathcal{L}^{-1}\left\{\frac{1}{s^{n}} \mathcal{L}\left\{R\left(\sum_{j=0}^{\infty} u_{j}\right)+N\left(\sum_{j=0}^{\infty} u_{j}\right)\right\}\right\}=f-\mathcal{L}^{-1}\left\{\frac{1}{s^{n}} \mathcal{L}\{R(u)+N(u)\}\right\}
\end{aligned}
$$

i.e $u=\sum_{i=0}^{\infty} u_{i}$ calculated according to (18) satisfies equation (13) and hence it is a solution to (6).

\section{Applications}

In this section, some examples will be introduced to illustrate the modified of NIM.

Example 4.1: Consider the following $3^{\text {rd }}$ order nonlinear homogeneous PDE:

$$
u_{t}+6 u^{2} u_{x}+u_{x x x}=0, \quad u(x, 0)=k \operatorname{sech}(k x)
$$

Then $L(u)=\frac{\partial u}{\partial t}$ i.e. $n=1, R(u)=u_{x x x}, N(u)=6 u^{2} u_{x}$ and since $g=0$ then: 


$$
\begin{aligned}
& u_{0}=f=f_{0}+\mathcal{L}^{-1}\left\{\frac{1}{s} \mathcal{L}\{g\}\right\}=k \operatorname{sech}(k x)+\mathcal{L}^{-1}\left\{\frac{1}{s} \mathcal{L}\{0\}\right\}=k \operatorname{sech}(k x) \\
& u_{1}=-\mathcal{L}^{-1}\left\{\frac{1}{s} \mathcal{L}\left\{R\left(u_{0}\right)+N\left(u_{0}\right)\right\}\right\}=-\mathcal{L}^{-1}\left\{\frac{1}{s} \mathcal{L}\{R(k \operatorname{sech}(k x))+N(k \operatorname{sech}(k x))\}\right\} \\
& =-\mathcal{L}^{-1}\left\{\frac{1}{s} \mathcal{L}\left\{k^{4} \tanh (k x)\left(-\operatorname{sech}(k x)+6 \operatorname{sech}^{3}(k x)\right)-6 k^{4} \tanh (k x) \operatorname{sech}^{3}(k x)\right\}\right\} \\
& =k^{4} \tanh (k x) \operatorname{sech}(k x) t \\
& u_{2}=-\mathcal{L}^{-1}\left\{\frac{1}{s} \mathcal{L}\left\{R\left(u_{1}\right)+N\left(u_{0}+u_{1}\right)-N\left(u_{0}\right)\right\}\right\} \\
& =-\mathcal{L}^{-1}\left\{\frac { 1 } { s } \mathcal { L } \left\{R\left(k^{4} \tanh (k x) \operatorname{sech}(k x) t\right)+N\left(k \operatorname{sech}(k x)+k^{4} \tanh (k x) \operatorname{sech}(k x) t\right)\right.\right. \\
& -N(k \operatorname{sech}(k x))\}\} \\
& =\frac{1}{2} k^{7} \tanh (k x)\left(1-2 \operatorname{sech}^{2}(k x)\right) t^{2}+k^{10} \tanh (k x)\left(6 \operatorname{sech}^{3}(k x)-10 \cosh ^{5}(k x)\right) t^{3} \\
& +k^{13}\left(3 \operatorname{sech}^{3}(k x)-9 \operatorname{sech}^{5}(k x)+6 \operatorname{sech}^{7}(k x)\right) t^{4}
\end{aligned}
$$

Then from (2), we have:

$$
\begin{aligned}
u(x, t)=u_{0}(x, t) & +u_{1}(x, t)+u_{2}(x, t)+\cdots \\
& =k \operatorname{sech}(k x)+k^{4} \tanh (k x) \operatorname{sech}(k x) t+\frac{1}{2} k^{7} \tanh (k x)\left(1-2 \operatorname{sech}^{2}(k x)\right) t^{2} \\
& +k^{10} \tanh (k x)\left(6 \operatorname{sech}^{3}(k x)-10 \cosh ^{5}(k x)\right) t^{3} \\
& +k^{13}\left(3 \operatorname{sech}^{3}(k x)-9 \operatorname{sech}^{5}(k x)+6 \operatorname{sech}^{7}(k x)\right) t^{4}+\cdots \\
& =k \operatorname{sech}(k x)+k^{4} \tanh (k x) \operatorname{sech}(k x) t+\frac{1}{2} k^{7} \tanh (k x)\left(1-2 \operatorname{sech}^{2}(k x)\right) t^{2} \\
& +\frac{1}{6} k^{10} \tanh (k x)\left(\operatorname{sech}(k x)-6 \operatorname{sech}^{3}(k x)\right) t^{3}+\cdots
\end{aligned}
$$

This is closed to the exact solution:

$$
u(x, t)=k \operatorname{sech}\left(k\left(x-k^{2} t\right)\right)
$$

Example 4.2: Consider the following $2^{\text {nd }}$ order nonlinear homogeneous PDE:

$$
u_{t t}-u+\frac{1}{4} u_{x}^{2}=0, \quad u(x, 0)=1+x^{2}, u_{t}(x, 0)=1
$$

Then $L(u)=\frac{\partial^{2} u}{\partial t^{2}}$ i.e. $n=2, R(u)=-u, N(u)=\frac{1}{4} u_{x}^{2}$ and since $g=0$ then:

$$
\begin{aligned}
u_{0}=f=f_{0}+f_{1} t+\mathcal{L}^{-1}\left\{\frac{1}{s^{2}} \mathcal{L}\{g\}\right\}=1+x^{2}+t+0=x^{2}+1+t \\
u_{1}=-\mathcal{L}^{-1}\left\{\frac{1}{s^{2}} \mathcal{L}\left\{R\left(u_{0}\right)+N\left(u_{0}\right)\right\}\right\}=-\mathcal{L}^{-1}\left\{\frac{1}{s^{2}} \mathcal{L}\left\{R\left(x^{2}+1+t\right)+N\left(x^{2}+1+t\right)\right\}\right\} \\
=-\mathcal{L}^{-1}\left\{\frac{1}{s^{2}} \mathcal{L}\left\{-x^{2}-1-t+\frac{1}{4}(2 x)^{2}\right\}\right\}=\mathcal{L}^{-1}\left\{\frac{1}{s^{2}} \mathcal{L}\{1+t\}\right\}=\frac{t^{2}}{2}+\frac{t^{3}}{6}=\frac{t^{2}}{2}+\frac{t^{3}}{3 !}
\end{aligned}
$$




$$
\begin{aligned}
u_{2}=-\mathcal{L}^{-1}\left\{\frac{1}{s^{2}} \mathcal{L}\right. & \left.\left\{R\left(u_{1}\right)+N\left(u_{0}+u_{1}\right)-N\left(u_{0}\right)\right\}\right\} \\
& =-\mathcal{L}^{-1}\left\{\frac{1}{s^{2}} \mathcal{L}\left\{R\left(\frac{1}{2} t^{2}+\frac{1}{6} t^{3}\right)+N\left(x^{2}+1+t+\frac{1}{2} t^{2}+\frac{1}{6} t^{3}\right)-\frac{1}{4}(2 x)^{2}\right\}\right\} \\
& =-\mathcal{L}^{-1}\left\{\frac{1}{s^{2}} \mathcal{L}\left\{-\frac{1}{2} t^{2}-\frac{1}{6} t^{3}+\frac{1}{4}(2 x)^{2}-\frac{1}{4}(2 x)^{2}\right\}\right\}=\frac{t^{4}}{24}+\frac{t^{5}}{120}=\frac{t^{4}}{4 !}+\frac{t^{5}}{5 !}
\end{aligned}
$$

$u_{3}=-\mathcal{L}^{-1}\left\{\frac{1}{s^{2}} \mathcal{L}\left\{R\left(u_{2}\right)+N\left(u_{0}+u_{1}+u_{2}\right)-N\left(u_{0}+u_{1}\right)\right\}\right\}=\frac{t^{6}}{720}+\frac{t^{7}}{5040}=\frac{t^{6}}{6 !}+\frac{t^{7}}{7 !}$

Then from (2), we have:

$$
u(x, t)=u_{0}(x, t)+u_{1}(x, t)+u_{2}(x, t)+\cdots=x^{2}+1+t+\frac{t^{2}}{2}+\frac{t^{3}}{3 !}+\frac{t^{4}}{4 !}+\frac{t^{5}}{5 !}+\frac{t^{6}}{6 !}+\frac{t^{7}}{7 !}+\cdots
$$

This is closed to the exact solution:

$$
u(x, t)=x^{2}+e^{t}
$$

Example 4.3: Consider the following 3rd order nonlinear inhomogeneous PDE:

$$
u_{t t t}+u^{2}-u u_{x}-u=3 e^{x+t}, \quad u(x, 0)=0, \quad u_{t}(x, 0)=e^{x}, \quad u_{t t}(x, 0)=2 e^{x}
$$

Then $L(u)=\frac{\partial^{3} u}{\partial t^{3}}$ i. e. $n=3, R(u)=-u, N(u)=u^{2}-u u_{x}$ and since $g=3 e^{x+t}$ then:

$$
\begin{gathered}
u_{0}=f=f_{0}+f_{1} t+f_{2} \frac{t^{2}}{2 !}+\mathcal{L}^{-1}\left\{\frac{1}{s^{2}} \mathcal{L}\{g\}\right\}=0+e^{x} t+e^{x} t^{2}+\mathcal{L}^{-1}\left\{\frac{1}{s^{2}} \mathcal{L}\left\{g 3 e^{x+t}\right\}\right\} \\
=e^{x} t+e^{x} t^{2}+\frac{3}{2} e^{x}\left(2 e^{t}-t^{2}-2 t-2\right)=e^{x}\left(3 e^{t}-3-2 t-\frac{t^{2}}{2}\right) \\
\begin{array}{c}
u_{1}=-\mathcal{L}^{-1}\left\{\frac{1}{s^{2}} \mathcal{L}\left\{R\left(u_{0}\right)+N\left(u_{0}\right)\right\}\right\}=-\mathcal{L}^{-1}\left\{\frac{1}{s^{2}} \mathcal{L}\left\{R\left(e^{x}\left(3 e^{t}-3-2 t-\frac{t^{2}}{2}\right)\right)+N\left(e^{x}\left(3 e^{t}-3-2 t-\frac{t^{2}}{2}\right)\right)\right\}\right\} \\
=e^{x}\left(3 e^{t}-3-3 t-\frac{3 t^{2}}{2}-\frac{t^{3}}{2}-\frac{t^{4}}{12}-\frac{t^{5}}{120}\right) \\
u_{2}=-\mathcal{L}^{-1}\left\{\frac{1}{s^{2}} \mathcal{L}\left\{R\left(u_{1}\right)+N\left(u_{0}+u_{1}\right)-N\left(u_{0}\right)\right\}\right\} \\
=e^{x}\left(3 e^{t}-3-3 t-\frac{3 t^{2}}{2}-\frac{t^{3}}{2}-\frac{t^{4}}{8}-\frac{t^{5}}{40}-\frac{t^{6}}{240}-\frac{t^{7}}{2520}-\frac{t^{8}}{40320}\right)
\end{array}
\end{gathered}
$$

Then from (2), we have: 


$$
\begin{aligned}
u(x, t)=u_{0}(x, t) & +u_{1}(x, t)+u_{2}(x, t)+\cdots= \\
& =e^{x}\left(9 e^{t}-9-8 t-\frac{7 t^{2}}{2 !}-\frac{6 t^{3}}{3 !}-\frac{5 t^{4}}{4 !}-\frac{4 t^{5}}{5 !}-\frac{3 t^{6}}{6 !}-\frac{2 t^{7}}{7 !}-\frac{t^{8}}{8 !}-\cdots\right) \\
& =e^{x}\left(9 e^{t}-9-8 t-\frac{7 t^{2}}{2 !}-\frac{6 t^{3}}{3 !}-\frac{5 t^{4}}{4 !}-\frac{4 t^{5}}{5 !}-\frac{3 t^{6}}{6 !}-\frac{2 t^{7}}{7 !}-\frac{t^{8}}{8 !}-\cdots\right. \\
& +\left[-t+t-\frac{2 t^{2}}{2 !}+\frac{2 t^{2}}{2 !}-\frac{3 t^{3}}{3 !}+\frac{3 t^{3}}{3 !}-\frac{4 t^{4}}{4 !}+\frac{4 t^{4}}{4 !}-\frac{5 t^{5}}{5 !}+\frac{5 t^{5}}{5 !}-\frac{6 t^{6}}{6 !}+\frac{6 t^{6}}{6 !}-\frac{7 t^{7}}{7 !}+\frac{7 t^{7}}{7 !}-\frac{8 t^{8}}{8 !}\right. \\
& \left.\left.+\frac{8 t^{8}}{8 !}-\cdots\right]\right) \\
& =e^{x}\left(9 e^{t}-9-9 t-\frac{9 t^{2}}{2 !}-\frac{9 t^{3}}{3 !}-\frac{9 t^{4}}{4 !}-\frac{9 t^{5}}{5 !}-\frac{9 t^{6}}{6 !}-\frac{9 t^{7}}{7 !}-\frac{9 t^{8}}{8 !}-\cdots\right. \\
& \left.+\left[t+t^{2}+\frac{t^{3}}{2 !}+\frac{t^{4}}{3 !}+\frac{t^{5}}{4 !}+\frac{t^{6}}{5 !}+\frac{t^{7}}{6 !}+\frac{t^{8}}{7 !}+\cdots\right]\right) \\
& =e^{x}\left(9 e^{t}-9\left[1+t+\frac{t^{2}}{2 !}+\frac{t^{3}}{3 !}+\frac{t^{4}}{4 !}+\frac{t^{5}}{5 !}+\frac{t^{6}}{6 !}+\frac{t^{7}}{7 !}+\frac{t^{8}}{8 !}+\cdots\right]\right. \\
& \left.+t\left[1+t+\frac{t^{2}}{2 !}+\frac{t^{3}}{3 !}+\frac{t^{4}}{4 !}+\frac{t^{5}}{5 !}+\frac{t^{6}}{6 !}+\frac{t^{7}}{7 !}+\cdots\right]\right)
\end{aligned}
$$

This is closed to the exact solution:

$$
u(x, t)=t e^{x+t}
$$

\section{Conclusion}

In this research, a new modification of the iterative method to solve PDEs is proposed. The experimental results show that the suggested modification is computationally efficient for solving non-linear, non-homogenous PDEs and can easily be implemented since it is free of using Adomian polynomials when dealing with the nonlinear terms like in the ADM and being free of using the Lagrange multiplier as in the VIM. The method proves to be simple in its principles and convenient for computer algorithms. It has also been witnessed that a few approximations can be used to achieve a high degree of accuracy.

\section{References}

1. Rach, R. (1987). On the Adomian (decomposition) method and comparisons with Picards method. Journal of Mathematical Analysis and Applications, 128(2), 480-483. DOI: 10.1016/0022247x(87)90199-5.

2. Hosseini, M., and Nasabzadeh, H. (2007). Modified Adomian decomposition method for specific second-order ordinary differential equations. Applied Mathematics and Computation, 186(1), 117123. DOI: 10.1016/j.amc.2006.07.094.

3. Abassy, T. A. (2010). Improved Adomian decomposition method. Computers and Mathematics with Applications, 59(1), 42-54. DOI: 10.1016/j.camwa.2009.06.009.

4. Al-Hayani, W. (2011). Adomian decomposition method with Green's function for sixth-order boundary value problems. Computers and Mathematics with Applications, 61(6), 1567-1575. DOI: 10.1016/j.camwa.2011.01.025.

5. Kareem, Z. H., and Tawfiq, L. N. M. (2020). Recent Modification of Decomposition Method for Solving Nonlinear Partial Differential Equations. Journal of Advances in Mathematics. 18, 2347-1921. DOI: https://doi.org/10.24297/jam.v18i.8744

6. Agom, E. U., and Ogunfiditimi, F. O. (2016). Numerical Application of Adomian Decomposition Method to One Dimensional Wave Equations. International Journal of Science and Research (IJSR), 5(5), 2306-2309. DOI: 10.21275/v5i5.nov162419.

7. Enadi, M. O., and Tawfiq, L.N.M. (2019). New Technique for Solving Autonomous Equations. Ibn AlHaitham Journal for Pure and Applied Science, 32(2), 123-130. DOI: 10.30526/32.2.2150.

8. He, J.-H. (2006). Homotopy perturbation method for solving boundary value problems. Physics Letters A, vol. 350(1-2), 87-88. DOI: 10.1016/j.physleta.2005.10.005. 
9. Jafari, H., and Saeidy, M. (2008). Application of Homotopy Perturbation Method for Solving Gas Dynamics Equation. Applied Mathematical Sciences, 2(48), 2393-2396. URL: http://www.mhikari.com/ams/amspassword-2008/ams-password45-48-2008/index.html.

10. Yu, J., and Huang, J.-G. (2010). Application of Homotopy Perturbation Method for the Reactiondiffusion Equation. International Journal of Nonlinear Sciences and Numerical Simulation, 11(Supplement). DOI: 10.1515/ijnsns.2010.11.s1.61.

11. Afrouzi, G., Ganji, D. D., Hosseinzadeh, H., and Talarposhti, R. (2011). Fourth Order Volterra Integrodifferential Equations Using Modified Homotopy-perturbation Method. Journal of Mathematics and Computer Science, 3(2), 179-191. DOI: 10.22436/jmcs.03.02.10.

12. Az-Zo'bi, E. (2012). Modified Laplace Decomposition Method. World Applied Sciences Journal, 18(11), 1481-1486. URL: https://www.idosi.org/wasj/wasj18(11)/1.pdf. DOI: 10.5829/idosi.wasj.2012.18.11.1522.

13. Osama H. Mohammed, Huda A. Salim. (2018). Computational methods based Laplace decomposition for solving nonlinear systems of fractional order differential equations. Alexandria Engineering Journal, 57(4), 3549-3557. DOI: 10.1016/j.aej.2017.11.020.

14. Momani, S., Odibat, Z., and Alawneh, A. (2007). Variational iteration method for solving the spaceand time-fractional KdV equation. Numerical Methods for Partial Differential Equations, 24(1), 262 271. DOI: $10.1002 /$ num.20247.

15. Salehpoor, E., and Jafari, H. (2011). Variational Iteration Method A Tools For Solving Partial Differential Equations. Journal of Mathematics and Computer Science, 2(2), 388-393. DOI: 10.22436/jmcs.002.02.18.

16. Wang, Q., and Fu, F. (2012). Variational Iteration Method for Solving Differential Equations with Piecewise Constant Arguments. International Journal of Engineering and Manufacturing, 2(2), 36-43. DOI: 10.5815/ijem.2012.02.06.

17. Tawfiq, L.N.M. (2016). Using collocation neural network to solve Eigenvalue problems. MJ Journal on Applied Mathematics, 1(1), 1-8. DOI:10.1155/2014/906376.

18. Salih H, Tawfiq LNM, Yahya ZRI, Zin S M. (2018). Solving Modified Regularized Long Wave Equation Using Collocation Method. Journal of Physics: Conference Series, 1003(012062), 1-10. DOI:10.1088/1742-6596/1003/1/012062.

19. Enadi MO, Tawfiq LNM. (2019). New Approach for Solving Three Dimensional Space Partial Differential Equation. Baghdad Science Journal, 16(3), 786-792. DOI:10.21123/bsj.2019.16.3(Suppl.).0786. II

http://bsj.uobaghdad.edu.iq/index.php/BSJ/article/view/4153.

20. Tawfiq, L.N.M.; Naoum, R.S. (2007). Density and approximation by using feed-forward Artificial neural networks. Ibn Al-Haitham Journal for Pure and Applied Sciences, 20(1), 67-81. URL: http://jih.uobaghdad.edu.iq/index.php/j/article/view/1335.

21. Effati, S., and Pakdaman, M. (2010). Artificial neural network approach for solving fuzzy differential equations. Information Sciences, 180(8), 1434-1457. DOI: 10.1016/j.ins.2009.12.016.

22. Tawfiq, L. N. M.; Oraibi, Y. A. (2013). Fast Training Algorithms for Feed Forward Neural Networks. Ibn Al-Haitham Journal for Pure and Applied Science, 26(1), 275-280. :URL: http://jih.uobaghdad.edu.iq/index.php/j/article/view/534.

23. Ali MH, Tawfiq LNM, Thirthar A. A. (2019). Designing Coupled Feed Forward Neural Network to Solve Fourth Order Singular Boundary Value Problem. Revista Aus, 26(2), 140-146.।I DOI: 10.4206/aus.2019.n26.2.20. URL: http://www.ausrevista.com/26-2.html.

24. Tawfiq, L.N.M., and Salih, O. M. (2019). Design neural network-based upon decomposition approach for solving reaction-diffusion equation. Journal of Physics: Conference Series, 1234(1234 012104), 1 8. DOI: 10.1088/1742-6596/1234/1/012104.

25. Gejji, V. D., and Jafari, H. (2006). An iterative method for solving non linear functional equations. J. Math. Anal. Appl. 316, 753-763. DOI:10.1016/j.jmaa.2005.05.009.

26. Bhalekar, S., and Gejji, V. D. (2008). New iterative method: Application to partial differential equations. Appl. Math. Comput, 203, 778-783. DOI:10.1016/j.amc.2008.05.071. 
27. Yaseen, M., and Samraiz, M. (2012). The Modified New Iterative Method for Solving Linear and Nonlinear Klein-Gordon Equations. Applied Mathematical Sciences, 6(60), 2979-2987. URL: http://www.m-hikari.com/ams/ams-2012/ams-57-60-2012/yaseenAMS57-60-2012.

28. Al-Subaihi, I.A. (2015). Three-Step Iterative Method for Solving Nonlinear Equations. Universal Journal of Applied Mathematics, 3(2): 29-33. DOI: 10.13189/ujam.2015.030204.

29. Nisse K, Nisse L. (2018). An Iterative Method for Solving a Class of Fractional Functional Differential Equations with "Maxima". Mathematics, 6(1). DOI:10.3390/math6010002.

30. Ucar, F., Yaman, V., and Yilmaz, B. (2018). Iterative Methods For Solving Nonlinear Lane-Emden Equations. Marmara Fen Bilimleri Dergisi, 30 (2). 176-188. DOI: 10.7240/marufbd.410960.

31. Cohen, A. M. (2007). Numerical methods for Laplace transform inversion. New York: Springer. ISBN: 978-0387282619. 